\title{
El poder de la primera persona
}

\author{
CARLOS PEREDA \\ Instituto de Investigaciones Filosóficas \\ Universidad Nacional Autónoma de México \\ jcarlos@filosoficas.unam.mx
}

Resumen: En este artículo se explora la pregunta "¿quién soy yo?" y qué
poder o poderes, si es que alguno, se le puede atribuir a la primera persona.
En primer lugar, se confrontan las posiciones de Descartes y de Hume, y
se considera como un posible desarrollo de la de Hume el eliminacionismo
biológico de la vida mental. Se introducen algunas razones para dudar de las
tres posiciones. En segundo lugar, se busca justificar tres posibles conjuntos
de poderes de la primera persona: la primera persona dispone de órganos
de los sentidos confiables y de usos de las palabras no menos confiables; la
primera persona es capaz de conocerse a sí misma; la primera persona posee
la capacidad de intervenir en el mundo: puede iniciar secuencias de sucesos.
Palabras clave: poder, primera persona, identidad, vértigos argumentativos

\begin{abstract}
This paper explores the question "who am I?" and what power or powers, if any, may be attributed to the first person. In the first place, the views of Descartes and Hume are contrasted and a biological eliminativism of mental life is considered as a possible development of the Humeian view. Several reasons are offered to cast doubt on all three positions. In the second place, an attempt is made to justify three possible sets of first-person powers: the first person is equipped with reliable sensory organs and with a no-less reliable use of words; the first person is capable of knowing him/herself; the first person has the capacity to intervene in the world: to initiate sequences of events.
\end{abstract}

Key words: power, first person, identity, argumentative vertigos

\section{El experimento de Hume}

Hume propuso un experimento: quien quiera descubrir quién es, que mire dentro de sí. Resultado: por más que me busco no me encuentro. Quien se examina fuera de sus percepciones, o de sus percepciones de percepciones, no encuentra, según el Tratado de la naturaleza humana, nada que posea la importancia de que presumen los usos militantes de la expresión "identidad personal":

cuando más íntimamente penetro en lo que llamo yo mismo, tropiezo siempre con una u otra percepción particular, de calor o frío, luz o sombra, amor u odio, dolor o placer. Nunca puedo aprehenderme yo mismo sin una percepción, y nunca puedo observar más que la percepción. ${ }^{1}$

${ }^{1}$ David Hume, A Treatise of Human Nature, p. 252. 
Con independencia de Hume este experimento continúa provocando muchas y hasta insospechadas alarmas: ¿tal vez no exista la tan alardeada identidad personal? En lugar de un yo, ¿sólo ocurren de vez en cuando algunas sensaciones y percepciones? No obstante, si no hay más que sensaciones y percepciones particulares, ¿de dónde proviene ese arraigado sentir que yo soy yo y nadie más, aunque tampoco menos que yo? Sobre todo, ¿cómo respaldo mi convicción de que tengo el poder de decidir qué hago o dejo de hacer con mi vida? Adelantándose a zozobras como éstas, en la Meditación segunda, Descartes propuso un yo hecho de una sustancia diferente de la sustancia material:

¿Qué soy, pues? Una cosa que piensa. ¿Qué es una cosa que piensa? Es una cosa que duda, entiende, concibe, afirma, niega, quiere, no quiere y, también, imagina y siente. ${ }^{2}$

Esta respuesta adquirió vasto prestigio. Sin embargo, ¿cómo algo no físico sería capaz de poseer efectos físicos? ${ }^{3}$ ¿No tendrá razón Hume y mi subjetividad se reduce al sucederse de sensaciones y percepciones? Sin embargo, afirmar que "tropiezo siempre con una u otra percepción particular", ¿no presupone que hay alguien que tropieza? La respuesta de Hume a su experimento, ¿no sugiere que tiene que haber un sujeto que percibe? ¿Con qué razones afirma Hume que no se encuentra y, a la vez, que él se topa con varias sensaciones y percepciones, y no con otras? Objeciones como éstas quizá no son graves. No es difícil reformular la respuesta de Hume con reportes impersonales como el siguiente: "Al investigar lo que se llama 'yo mismo' no se encontraron más que sensaciones y percepciones en flujo constante". Ese reporte reduccionista o, radicalizándolo más allá de Hume, eliminador, ${ }^{4}$ podría situarse como perteneciente a la clase de reportes científicos a la que

${ }^{2}$ R. Descartes, Meditación segunda.

${ }^{3}$ En una carta a Descartes, la princesa Elizabeth ya observó que "sería más fácil para mí atribuir materia y extensión al alma, que atribuirle a un cuerpo inmaterial la capacidad de mover y ser movido por un cuerpo", en Descartes: Philosophical Letters, p. 140.

${ }^{4}$ El eliminacionismo es una posición reciente; los atomistas griegos como Leucipo y Demócrito intentaron explicar las propiedades mentales a partir de su metafísica atomista, pero sin negarlas. Algunas tempranas propuestas de eliminacionismo se encuentran en P. Feyerabend, "Mental Events and the Brain", y R. Rorty, "MindBody Identity, Privacy, and Categories." Proponentes recientes, y tal vez más radicales de esta posición, son: Paul Churchland, "Eliminative Materialism and the Propositional Attitudes", Patricia Churchland, Neurophilosophy, y Stephen Stich, From Folk Psychology to Cognitive Science: The Case Against Belief.

Diánoia, vol. LI, no. 57 (noviembre 2006). 
pertenece el siguiente: "Al investigar lo que se llamó 'flogisto', no se encontraron más que procesos de combustión y oxígeno."

Entonces, todo aquello a que se hace alusión con palabras como "identidad personal", "subjetividad", "yo" o, si se prefiere, "la actitud en primera persona", sus "deseos" y "creencias", sus "angustias" y "alegrías" por lo que le sucede, le sucedió, le sucederá, sus "acciones"... ¿no son más que fantasías, productos de una psicología precientífica, tan equivocadas como esa otra fantasía de la química previa a Lavoisier, el flogisto? Recuerdo que hace unos días nos juntamos varios vecinos para averiguar la causa de una reciente humedad en los departamentos. No sin insolencia pregunté: "¿Quién ha sido el irresponsable que deja abierta las llaves del agua?" Con mucha vergüenza, descubrí que yo era ese irresponsable que, desde hace dos días, no había cerrado por completo la llave de un baño que rara vez usamos.

En tales circunstancias, de seguro nadie se excusaría con una forma mixta de comunicación como: "La descuidada secuencia de moléculas que dejó abierta la llave del baño ha dejado de existir. En su lugar ahora sabemos del movimiento de moléculas que atraviesan, de manera organizada y perfectamente causal, las membranas celulares de neuronas estructuradas en redes complejas en las cuales ciertas conexiones y rutas, aunque bastante estables, son, aun como parte de un cerebro adulto, dinámicamente reforzadas mientras que otras son debilitadas." En estas hipotéticas disculpas se trata de "formas heterogéneas" de comunicación, porque se combina el punto de vista de la primera persona en cuanto agente práctico (presupuesto en calificativos como "descuidada"...) con explicaciones que aluden a saberes ("moléculas"..., "neuronas"...) desde el punto de vista de la tercera persona.

No obstante, esas hipotéticas prácticas de comunicación ("la descuidada secuencia de moléculas...") ¿no se encuentran más cerca de hábitos comunes de lo que solemos confesarnos? Se da unos pasos en esa dirección cuando ofrecemos disculpas heterogéneas como: "Lamento mis modales de ayer. Había comido puerco y, ya sabes, la digestión lenta tiende a volverme irritable." Tampoco es raro que procuremos defendernos de nosotros mismos con precauciones heterogéneas del tipo: "No tomaré ni un vaso de cerveza porque tengo todavía dos horas de trabajo por delante." También damos y recibimos consejos heterogéneos como: "Tome mucho líquido, vitamina $\mathrm{C}$ y olvídese de sus preocupaciones en el trabajo."

Disculpas heterogéneas, precauciones heterogéneas, consejos heterogéneos... ¿acaso no hay que reconstruir todas esas formas de expresarnos como posibles síntomas de un determinismo biológico? 
Objeción: en las más diversas prácticas sólo nos atrevemos a avanzar menos de medio camino en la dirección que señalan esas formas heterogéneas de comunicación. Más todavía, ¿por qué a menudo se sospecha que avanzar más en una de esas direcciones implicaría sucumbir en un vértigo simplificador en los razonamientos? Después de todo, nos hemos resignado a que la tierra se mueva alrededor del Sol y no al revés. ¿Podremos algún día aprender a vivir no sólo sin percepciones, deseos, creencias, emociones, expectativas, sino también sin sus portadores y, así, sin actitudes en primera persona y sin acciones? De seguro —iobtusamente? - muchos gritarán: "No". Tal vez se apoye esa terca convicción, insistiendo a su vez: ¿quiénes serían esos que "aprenderían a vivir"?

Para terciar en estas resbaladizas zozobras, quiero explorar la siguiente propuesta, o esbozo de propuesta: de seguro con el pronombre de la primera persona no se hace referencia a una "cosa" hecha de sustancia pensante, como propone Descartes. No obstante, las secuencias de percepciones, y se puede agregar, y de deseos, creencias, emociones, expectativas..., tampoco meramente se suceden, como sugiere Hume. En ese sucederse se van conformando, entre otros, el poder de producir actitudes en primera persona, al constituirse en un cuerpo, y sólo un cuerpo, algunos modos de seguir y de seguirse de una experiencia a otra, y de seguir y de seguirse de un suceso al otro que la actitud en primera persona produce y califica como "sus acciones".

La actitud en primera persona postula, pues, como primer conjunto de propiedades, órganos de los sentidos y uso de las palabras confiables. Segundo conjunto de propiedades: la primera persona cree disponer de una capacidad de conocerse y de adoptar diversas posiciones sobre el mundo y, de manera peculiar, sobre esa parte del mundo que es ella misma. Tercer conjunto de propiedades: la primera persona también presume que es capaz no sólo de observar sucesos, sino también de producirlos.

En cualquier caso, seguir y seguirme de una experiencia a otra no solamente proporciona un acceso a mi pasado, al menos, a fragmentos de él, dándome una duración en el tiempo. Esto es verdad. Sin embargo, me puedo considerar a mí mismo que estoy durando porque al experimentar y actuar dispongo de una compleja capacidad, a la vez, cualitativa y autorreconocedora. Entre otras razones, ese seguirme en experiencias y acciones me permite suponer que yo existía en los intervalos en los que no recuerdo o estoy dormido. Así, aprendo a usar, entre otras palabras, el pronombre "yo". 
No obstante, esta capacidad que sobreviene a experiencias y acciones - ¿ io es causada por ellas, o es función, o emerge de ellas...?y me constituye, no es un coágulo de "sustancia pensante". La unidad del seguimiento no proviene de algún núcleo recóndito y fijo, sino que se lleva a cabo con diversos traslapes, a menudo con férreos traslapes que interconectan cualitativamente, y con frecuencia también con algún grado de autorreconocimiento, experiencias y acciones. A su vez, estas interconexiones permiten formular metainducciones con las que continúo siguiéndome si no recuerdo o estoy dormido, o me rastreo, en ocasiones con desesperación, de una experiencia a otra que no deseo (como cuando me acosan dolores físicos que no controlo).

$¿$ Hay, entonces, actitud en primera persona porque en determinados organismos se constituyen poderes, ciertas capacidades? El modo humano de sentir y actuar no se lleva a cabo a solas, sino en interrelación con otros animales humanos. Por lo pronto, parece difícil que un animal humano pueda seguirse sin palabras. Pero las palabras no las inventa cada individuo cada vez. Son productos de la fábrica social y de sus historias: han sido, son, de todas, de todos.

Sin embargo, las raíces de la sociabilidad y los procesos de identificación y contraidentificación son de seguro más básicos que el lenguaje. ¿Acaso en cada animal humano no se encuentran ya en los mecanismos de imitación del comportamiento de los demás animales humanos? Entre otros, sus primeros movimientos corporales y los procesos más elementales de comprensión y de aprendizaje quizá dependen de estos mecanismos. Por ejemplo, cuando los niños perciben a otros animales humanos actuar de la misma manera que ellos, ¿no infieren inmediatamente que esos otros animales poseen estados mentales similares a los propios? (Tal vez los procesos imitativos ni siquiera se reduzcan a imitar lo que los otros animales humanos hacen, sus conductas, sino también lo que tratan de hacer.)

Sin duda, estas incipientes capacidades son reforzadas con la aparición de procesos complejos de imitación que incluyen la simulación $\mathrm{y}$, por supuesto, la adquisición de una lengua. No obstante, aspectos básicos de la adquisición de lenguaje continúan dependiendo de la imitación; por ejemplo, el aprendizaje fonético y la estructura prosódica de cada lengua.

La expresión "adquisición de una lengua" recuerda ya que todas y todos aprendemos una lengua, y no directamente el lenguaje. Una lengua evoca tradiciones específicas, grupos, instituciones, hasta individuos. A su vez, dentro de una lengua, muchas palabras marcan mi seguir y seguirme, lo particularizan y, a menudo, lo enfrentan a otras tradiciones, 
grupos, instituciones, individuos. De esta manera, imitando y contraimitando, y en medio de palabras que corrigen y defienden percepciones, deseos, acciones..., los animales humanos instauran prácticas de reconocimiento y desconocimiento. Por otra parte, estas prácticas a menudo cobran dinámicas sociales -independientes de las intenciones de cualquier animal humano- que incluyen dispositivos reafirmadores de esas dinámicas.

En consecuencia, imitando, simulando y, muy pronto, usando palabras y otras herramientas, el animal humano se identifica y afilia a diversos animales humanos, sucesos, tiempos, lugares, y también se contraidentifica, no menos se separa de otros animales humanos. De esta manera, afiliaciones y resistencias suelen resultar comienzos de historias en las que el "yo" de "aquí" y "ahora" hace alianzas con el "ustedes" de "aquí" y "antes", y también se opone a el "él/ella" de "allá" y "ahora"... Por eso, no sólo como los otros cuerpos, los humanos somos animales en el espacio, y como los otros cuerpos vivos, sentimos, tenemos experiencias. También somos animales que, imitando a otros animales humanos, aprendemos a seguirnos en el tiempo y disponemos del poder de actuar con palabras y de servirnos desde técnicas tan simples como aquellas que prolongan el cuerpo humano -el martillo, el arado...hasta aquellas otras tan complejas como las biotecnologías y la red.

Quien afirma que afiliándonos y resistiéndonos organizamos los desplazamientos sociales de un lugar a otro, y el fluir temporal, narrando y narrándome, suscita elaboraciones como "nos reconocemos socialmente siguiéndonos en el pasado, más o menos integrándonos hasta el momento en que nos encontramos ahora". No sólo. Cualquier capacidad de seguimiento trata las experiencias y las acciones como si se extendieran hacia atrás, y también hacia adelante, en el futuro. En los seguimientos hacia el pasado, se recogen historias o esbozos de historias de la primera persona, pero también de la segunda y de la tercera, del singular y del plural. Cuando hay seguimientos en el presente y en el futuro solemos vivirnos, y describirnos, como si siguiéramos anticipaciones o hiciéramos planes, proyectos de la primera persona, pero también de la segunda y la tercera, del singular y del plural. Por eso podemos articular a los poderes que implican estos modos normativos de discontinuo seguir y seguirme con identificaciones y contraidentificaciones sociales en el pasado como una capacidad de seguimiento narrativo ${ }^{5}$ y aquellos

${ }^{5}$ La expresión "capacidad de seguimiento narrativo" puede tener un sentido descriptivo y uno normativo, o una mezcla de ambos. Defensas de una interrelación de ambos sentidos las encontramos en A. MacIntyre, After Virtue; C. Taylor, Sources of the Self; Paul Ricœur, Soi-même comme un autre.

Diánoia, vol. LI, no. 57 (noviembre 2006). 
que se orientan hacia el presente y el futuro como una capacidad de seguimiento planificador. Por supuesto, con expresiones como "seguimiento narrativo" y "seguimiento planeador" se hace referencia a varios aspectos de los mismos poderes, aspectos que a menudo interactúan.

iAh!... —se exclamará no sin decepción- pero ¿sólo porque dispongo de los poderes de seguir y de seguirme, en parte imitativamente, en medio de emocionadas experiencias en las que mi cuerpo a veces parece apoyarme y otras me interrumpe, y actuando con palabras y otras muchas herramientas en espacios y tiempos discontinuos y que, a menudo, se me imponen o se me escapan, soy? ¿Soy, entonces, porque mi cuerpo produce con identificaciones y contraidentificaciones sociales una primera persona que se narra no pocas veces con conflictos desde el pasado, y planea, a menudo con desarrolladas tecnologías y no menos conflictos, el presente y el futuro?

\section{Tres tipos de razonamientos vertiginosos}

Abruptamente retomo el experimento de Hume acerca de quién soy, dando unos pasos más allá de su posición deflacionista. Por lo pronto, modifico el punto de vista. En lugar de buscarme a mí mismo en actitud de primera persona, reformulo una versión de ese experimento desde el punto de vista de la tercera persona. Reiteremos, pues, la pregunta acerca de qué queda si se eliminan ya no las sensaciones y percepciones, sino los estados corporales y los roles sociales. Casi diría, lamentablemente, la respuesta a este experimento en tercera persona radicaliza la respuesta obtenida en primera persona: "Al investigar lo que se llama 'yo mismo' no se encuentran más que estados fisiológicos de un organismo y/o identificaciones y contraidentificaciones conformando roles sociales."

Así, a partir de perspectivas metodológicas que adoptan en exclusiva el punto de vista de la tercera persona, y buscan respaldarse en el desarrollo de investigaciones científicas, no sólo se han defendido y se defienden propuestas eliminadoras o reduccionistas acerca de quién soy, sino que con trabajados argumentos, se las magnifica en dos influyentes tradiciones de pensamiento que reafirman las alarmas del determinismo. Por doquier encontramos sus ecos.

Por un lado, vertiginosamente se sugiere que palabras como "identidad personal", "yo", "sujeto", "subjetividad", "poder de la primera persona" y hasta "percepciones", "deseos", "creencias", "emociones", "expectativas", "acciones" o "portador de percepciones, deseos, creencias, emociones, expectativas..." no son más que fragmentos del tosco 
vocabulario de una psicología, aunque todavía inmensamente popular, sin ninguna legitimidad científica. Tarde o temprano el desarrollo de las ciencias naturales eliminará ese primitivo modo de expresión, como ha eliminado tantos otros; lo sustituirá por uno más acorde a los rigurosos saberes de la neurofisiología, e incluso de la química, de la física.

Por otro lado, a partir de lo que propongo describir como un vértigo simplificador opuesto, aunque no menos influyente, también se reduce el significado de palabras como "identidad personal", "yo", "sujeto", "subjetividad"... a meras identificaciones y contra-identificaciones culturales, políticas, económicas, legales... De esta manera, la expresión "poder de la primera persona" sólo hace referencia a efectos - iinterpelaciones? - de algún sistema social.

Algunas consecuencias de estos razonamientos vertiginosos son previsibles. A partir de estos vértigos, por ejemplo, la ruidosa algarabía en torno a eso que los animales humanos califican como su "subjetividad" se evapora en fantasías más o menos sentimentales que producen, para su propio funcionamiento, algunos sistemas físicos, químicos, biológicos, psicológicos, sociales. Así, quien prosiga alguno de estos dos tipos de razonamiento, o una combinación de ambos, tal vez busque traducir el mandato "conócete a ti mismo" del oráculo de Delfos como: "toma cursos en neurofisiología, y química y física" y/o "aprende ciencias sociales".

¿Bromeo? Incluso quien se resista a esta traducción del oráculo porque la considera presa de perspectivas metodológicas que conducen a vértigos, con dificultad dejará de reconocer que se trata de vértigos del razonamiento que deforman o simplifican verdades. Después de todo, muy parcial —es el calificativo más débil que se me ocurre- sería el conocimiento que un animal humano posee de sí mismo si procura ocultar los sistemas físicos, químicos, biológicos, psicológicos, sociales... que lo producen o, al menos, contribuyen a hacerlo.

Sin embargo, ¿no hay que complementar las teorías, naturales y sociales, con una perspectiva metodológica capaz de recoger materiales provenientes de ese poder de la primera persona? Ante todo quiero explorar un poco el - ihipotético? - poder de la primera persona revisando sus tres conjuntos de postuladas propiedades. Primer conjunto: la primera persona dispone de órganos de los sentidos confiables y de usos de las palabras no menos confiables. Segundo conjunto: la primera persona es capaz de conocerse a sí misma y adoptar diversas posiciones sobre el mundo y, de manera peculiar, sobre esa parte del mundo que es ella misma. Tercer conjunto: la primera persona posee la no menos rara posibilidad de intervenir en el mundo: de iniciar secuencias de sucesos 
y de seguirse en esos sucesos. Este seguimiento convierte a tales sucesos en acciones propias de un yo. ${ }^{6}$

¿Cómo se justifica este primer conjunto de propiedades? Ha habido creencias que estaban fuertemente presupuestas en el conjunto de las creencias de una época y que, en algún momento, resultaron falsas. De manera similar, ¿no podría resultar que estas propiedades también sean falsas? Quizá no es verdad que, respecto de sus percepciones y uso de las palabras, la primera persona sólo a veces no sepa, se equivoque o engañe a otros o a sí misma. Tal vez sin cesar no sabe, se equivoca, engaña o se engaña. Entre otras razones, téngase en cuenta que los significados de las palabras en gran medida no dependen del control de alguna primera persona en particular. Además, como los animales humanos a menudo no sólo expresan sino que, antes todavía, articulan sus perspectivas con palabras, en consecuencia, lo que aportan sus percepciones, creencias, recuerdos, tampoco se encuentra por entero bajo su control. ${ }^{7}$

Es común encontrarse en circunstancias análogas con consecuencias graves para la perspectiva de la verdad como cuando se usan palabras acerca de las cuales una ciencia ha modificado el conocimiento que se dispone de sus referentes y se ignoran esos cambios. (Téngase en cuenta el uso tan corriente de palabras como "queso", "carne de res", "pescado", "espinaca"... En algún momento tal vez dejemos de clasificar los tipos de quesos de acuerdo con criterios gastronómicos — camembert, manchego, panela...- y comencemos a interesarnos por criterios que presuponen saberes médicos, como quesos que aumentan el colesterol y quesos que no lo hacen.) Pero si factores externos (el mundo y uno de sus fragmentos, la lengua) determinan el contenido de las creencias, entre ellas, de las creencias acerca de cómo hay que usar las palabras, entonces, tenemos que admitir que la primera persona desconoce parte de los contenidos que ella misma tiene acerca de qué habla y de sus correspondientes experiencias.

$\mathrm{Si}$ a este primer tipo de errores y de conocimientos parciales sobre la referencia de las palabras se le agregan los casos de engaños sobre la naturaleza o sobre algunos de los subsistemas del sistema social produ-

${ }^{6}$ David J. Chalmers en su libro The Conscious Mind: In Search of a Fundamental Theory, ha observado que el "hard problem" de la conciencia es el problema de la experiencia: de las cualidades fenoménicas o qualia. En este trabajo se intenta mostrar que en esta trama de dificultades hay dos problemas igualmente "hard" e interconectados: el problema de la experiencia y el problema de la acción.

${ }^{7}$ La posición externalista se ha defendido de varias maneras. Una temprana defensa la encontramos en H. Putnam, "The Meaning of 'Meaning'". 
cidos por una segunda o tercera persona, del singular o del plural, la situación se hace aún más difícil para propiedades que postulan la confiabilidad de los reportes de los órganos de los sentidos y de los usos de las palabras. Recordemos la diaria propaganda en los medios masivos de comunicación.

No obstante, demos todavía un paso más. A las equivocaciones, conocimientos parciales y engaños agreguemos la infatigable capacidad de engañarse a uno mismo en relación con el funcionamiento tanto de la naturaleza como de la sociedad y de la vida psicológica. A estas alturas, ¿no desaparece ya todo apoyo respecto de esta primera propiedad del poder de la primera persona?

Sin embargo, como primera persona, ¿puedo adoptar como regla práctica para comportarme que en mis percepciones y creencias, entre otras, mis creencias sobre los usos de las palabras constantemente están erradas? Abracemos una posición bastante extrema: aceptemos que con frecuencia la primera persona se desvía de la perspectiva de la verdad (equivocación, conocimiento parcial, engaño, engaño de sí misma). No obstante, a la vez, ¿no se tiene que insistir en que se trata de desviaciones? Debemos hacerlo si al menos defendemos la confiabilidad de los órganos de los sentidos y de los usos de las palabras en cuanto presunción. Como hacemos con respecto de cualquier regla de presunción, quien dude o sospeche en contra, en cada caso tendrá que ofrecer razones concretas para atacar en particular esa percepción, esa creencia, esa palabra. ¿Qué respalda a esa regla de presunción?

La respuesta es más simple de lo que se podría esperar (al menos, de lo que a menudo se espera): nos regimos según esa regla de presunción, vivimos de acuerdo con ella, porque en las más diversas prácticas -incluyendo las prácticas teóricas- no tenemos alternativa. Obsérvese que a menos que haya presunción de que la primera persona percibe, cree y habla correctamente y que, en consecuencia, la segunda o la tercera persona (o asumir el punto de vista de la segunda o de la tercera persona) que duda o sospecha tienen la carga de la prueba, no sólo seríamos por completo extraños a nosotros mismos - literalmente desconocidos-, sino que ni siquiera se podría comenzar a dudar o a sospechar. ¿Por qué?

Toda segunda o tercera persona es también una primera persona. Por lo tanto, si no se parte de la perspectiva metodológica de que la primera persona sabe en principio qué experimenta y cómo usa las palabras, nadie podría saber nunca qué se percibe, se cree, se recuerda, se habla. Nadie podría seguir nunca sus experiencias y seguirse a sí mismo a partir de ellas. La duda o la sospecha no encontraría ningún obje- 
to -ninguna aserción, ninguna orden, ningún presupuesto...- para ejercerse. Así, incluso faltarían las palabras para formular el menor atisbo de duda o de sospecha. Por lo tanto, para que esas tareas preciosas, dudar y sospechar, sean posibles, hay que presuponer la regla sobre la confiabilidad en principio de los reportes de los órganos de los sentidos y acerca del uso de las palabras.

Junto a este argumento general, también se podrían ofrecer algunos argumentos particulares. Si cierta cantidad de los teoremas que supongo que son pertenecientes al castellano fuesen del tipo "crepúsculo significa rebozo", nadie hubiese aprendido castellano, pues a partir de tales teoremas no se podrían tener relaciones complejas con los nativos de esa lengua. Sin embargo, ¿acaso algún animal humano podría "aprender" de manera sistemáticamente equivocada la lengua de sus interacciones prácticas más elementales: su "lengua materna"?

Segundo conjunto: la primera persona es capaz de conocerse a sí misma y de desdoblarse adoptando diversas posiciones sobre el mundo $\mathrm{y}$, de manera peculiar, sobre esa parte del mundo que es ella misma. Por lo pronto, con frecuencia, para seguir qué experimentan y cómo usan sus palabras la segunda y la tercera persona, del singular y del plural, se recogen varias clases de datos empíricos y se llevan a cabo inferencias a partir de sus conductas. A la vez, no es menos común dar por sentado que, respecto de sí misma, la primera persona prescinde de esos esfuerzos. Hasta se tiene la presunción de que la primera persona posee una relación peculiar con sus percepciones, deseos, creencias... ¿Qué significa "de manera peculiar"?

"Peculiar": al aspecto fenoménico de la experiencia no se puede acceder más que a partir del punto de vista de la primera persona; sin embargo, esas variadas propiedades ¿qué tipos de aportaciones, si es que alguno, proporcionan? Porque incluso si se acepta describir una longitud de onda como sonidos agudos, cada uno de esos modos de presentación ¿no implica conceptos diferentes?

Pero quizá los tres conjuntos de propiedades que constituyen, o contribuyen a constituir el poder de la primera persona - si es que lo hacen-, conforman subconjuntos de un conjunto. Conjetura: los aspectos cualitativos son base o contribuyen a conformar la base de la mayoría, si es que no de todas, nuestras primeras acciones. Tal vez no sólo eso. A lo largo de la vida, estos aspectos cualitativos continúan ofreciéndome las coordenadas para, valorando de inmediato, reaccionar: la sensación de dolor me lleva a quitar la mano del fuego; sentir frío me hace buscar resguardo; percibir un mal olor hace que me aleje 
de lo que lo produce; descubrir en la multitud a Pablo me hace proferir la oración "Acabo de ver a Pablo".

Sin embargo, ¿cómo puedo respaldar esta confianza de la primera persona respecto de la capacidad de conocerse a sí misma y de desdoblarse y modificarse y corregirse? De nuevo, como respecto del primer conjunto de propiedades, no tenemos otra alternativa práctica que considerar que este segundo conjunto se rige por una regla de presunción.

Si discutí algunas razones para restringir o eliminar la validez en principio de los dos primeros conjuntos de propiedades que podrían constituir el poder de la primera persona, el otro conjunto o, más bien, subconjunto de propiedades del poder de la primera persona, las condiciones de la acción, han sido y son mucho más atacadas. Como enseñan, por un lado, la bioquímica y las neurociencias, y, por otro, la antropología y las demás ciencias sociales, para no hablar del factor "contingencia" o, si se prefiere, de la suerte, buena o mala, la hechura del animal humano no está bajo el control de la primera persona. Por eso, tal vez somos incapaces de ser diferentes de quienes somos. $\mathrm{O}$, con mayor precisión: tal vez somos incapaces de ser diferentes de como naturalmente nos han hecho y socialmente nos rehacen. En consecuencia, somos incapaces de actuar diferente o, si se prefiere, de reaccionar diferente de como lo hacemos. Teniendo en cuenta algunas de estas razones, ¿qué se puede responder a quien observa que la producción de las acciones no depende de la primera persona, sino de antecedentes ajenos a sus decisiones?

Otra vez, en contra de estas amenazas en relación con el tercer subconjunto de propiedades o condición de la primera persona como agente, encontramos una regla de presunción igual de simple y similar a la que rige al primero y al segundo subconjuntos. Respecto de su adopción no tenemos alternativa. En cuanto primeras personas, no podemos siquiera imaginarnos sin capacidad de actuar. ¿Qué significa en este contexto de discusión "no podemos"? Además, ¿por qué "no podemos"? Estoy trabajando en la oficina y, de pronto, me siento cansado. Suspendo por eso mi trabajo y voy por un vaso de agua. O tal vez me ordene que tengo que acabar con este trabajo en la próxima media hora y, así, que debo aguantar sentado hasta que lo termine. Suponiendo que no se trata de un cansancio extremo, hasta sería difícil siquiera entender la sospecha de que no ha sido la primera persona la que ha decidido interrumpir su trabajo o continuarlo. ¿Quién podría haber sido sino ella? Éste es el tipo de circunstancias con las que, en cuanto primera persona, me enfrento día a día. Por eso no puedo dejar de tener como presunción el poder de la primera persona cuando procuro dar cuenta de él, pues 
si no tenemos alternativa a las reglas acerca de los saberes cualitativos en principio inmediatos de la primera persona y sobre su capacidad de actuar, ¿cómo se podría no partir de estas presunciones? Por otra parte (pero esta pregunta apela a saberes empíricos más complicados de justificar), si estas presunciones no fuesen correctas, la especie, ¿acaso hubiera sobrevivido?

No obstante, ¿qué estoy realmente afirmando, o al menos sugiriendo, con estos argumentos para respaldar el poder de la primera persona? $¿$ No regreso a recoger la arrogante bandera de que los animales humanos estamos "condenados a la acción"? La palabra "condena" sugiere desesperación. Quien recibe una condena suele desesperarse; sin embargo, a pesar del patetismo de la retórica (que en la situación anterior, por ejemplo, conduce a declarar que estoy "condenado" a "decidir con desesperación" si continuo sentado o camino por un vaso de agua), esa bandera cobija una verdad. Y aunque esa verdad se presta a fanfarrias que a menudo no son más que malentendidos, es una verdad. Pues, ¿cómo se podría evitar en actitud de primera persona no elegir, no actuar? Se conoce: quien en actitud de primera persona se hace a un lado para omitir tomar una decisión, también aprueba o desaprueba y se sigue a sí mismo, aprobándose o desaprobándose.

No hay escape. Desde el punto de vista de la primera persona no se puede suspender actuar bajo el pretexto de que las elecciones se encuentran predeterminadas, sea como resultado de la herencia biológica o de sistemas culturales, políticos, legales, económicos, articulados en la tradición y en las instituciones de un sistema social. Pero, otra vez, cuidado; pues cierto entusiasmo ya se enreda peligrosamente en las palabras, y ese entusiasmo invita a abrazar una perspectiva metodológica para comprender y explicar presa de un nuevo vértigo simplificador, el vértigo de la primera persona: aquel que convocan los usos militantes de la expresión "identidad personal" y expresiones exaltadoras como "estamos condenados a la acción". Con razón se insistirá: ¿con base en qué argumentos se elimina la sospecha de que cuando un animal humano "actúa" sólo operan ciertos sistemas biológicos o sociales? Tal vez la expresión "razones para actuar" de la primera persona no hace referencia más que a meras "racionalizaciones": pseudorazones para respaldar conductas que, de todas maneras, esa persona llevaría a cabo.

Imaginemos la siguiente estadística. Según ésta, los habitantes de América Latina en el $99 \%$ de las ocasiones tienen precio y aceptan corromperse. Tomando esa estadística como premisa mayor, razono: Yo soy latinoamericano. Yo he sido sobornado con el precio correcto. Por 
lo tanto, yo planeo corromperme. ¿Cómo hay que evaluar este argumento?

Más allá del estereotipo que expresa su —de seguro- falsa premisa mayor, el argumento puede interpretarse como argumento ambiguo, e incluso respaldado en una confusión significativa. Si la interpretación literal de la conclusión es justa, su formulación se encuentra en primera persona. En ese caso se trata, por varias razones, de un mal argumento. Desde el punto de vista de la primera persona, yo tengo que planear qué haré, qué curso de acción será el mío. Es mi integridad personal la que se encuentra en juego, pues planear y, así, planearme es una de las propiedades de la actitud en primera persona. Por eso, los sucesos que produzca serán mis acciones y yo seré responsable de ellas. De ahí que si se me reprocha inmoralidad y justifico mi decisión aludiendo a una estadística según la cual en el $99 \%$ de los casos las personas de mi entorno poseen esos usos y costumbres, con razón tal respuesta será juzgada como una excusa indefendible: pseudorazones. Entre otras acusaciones, se la criticará alegando que yo sé qué significa la palabra "soborno" y que está en mi poder actuar de otra manera: que ésta es, precisamente, una propiedad del poder de la primera persona.

Entonces, ¿por qué afirmé que el argumento puede interpretarse como argumento ambiguo y no como un mal argumento? Tal vez la formulación de la conclusión no es literal. Quizá en la conclusión no se busca establecer una decisión, sino una predicción. Se indicó: anticipar sucesos, predecir, es una función respecto del futuro del punto de vista de la tercera persona. Si se parte de una premisa que establece cómo se ha actuado en el $99 \%$ de los casos, desde el punto de vista de la tercera persona, salvo que haya una razón particular en contra, es correcto predecir que en el siguiente caso se continuará actuando de esa manera.

Sin embargo, también afirmé que el argumento podía interpretarse como si estuviera respaldado en una confusión significativa. "¿Quién soy yo?", “¿qué debo hacer con mi vida?”, son preguntas que atañen a las capacidades de seguimiento narrativo y planeador, que son aspectos de la actitud en primera persona. No obstante, el punto de vista de la primera persona y su creencia de que depende del yo qué clase de persona el yo planea ser entra en conflicto con los saberes desde el punto de vista de la tercera persona. Según esos saberes, nos encontramos "condenados" a las leyes, regularidades y tendencias, conocidas y desconocidas, que rigen el mundo natural, psicológico y social, histórico; además de la suerte, buena o mala. En este caso, ¿cómo puedo conciliar 
o, al menos, tomar en serio, los saberes del punto de vista de la primera y de la tercera persona?

Por lo pronto, se conoce que los diversos puntos de vista conforman posibilidades de la misma persona. Quien no se decida a encerrarse, pues, en una ceguera del mundo, incluyendo esa parte del mundo que conforman sus propias posibilidades, tendrá que procurar complementar los diversos puntos de vista o hacerlos competir y corregir el uno con los otros. Recuérdense las disculpas heterogéneas, las cautelas heterogéneas, los consejos heterogéneos. A la vez, no se olviden las resistencias, bastante generalizadas, a aceptar que se lleven demasiado lejos ese tipo de disculpas, cautelas, consejos.

\section{Conceptos inestables}

¿En qué consistiría llevar las formas mixtas de comunicación "demasiado lejos"? Lo haríamos dejando de complementar, o de matizar, el punto de vista de la primera persona en cuanto agente, para sustituirlo por entero por los saberes empíricos —sociales, psicológicos, biológicos, químicos...- - de la tercera persona. Por eso, la confusión entre decidir y predecir es significativa. Por eso, conceptos como animal humano, acción, deseo, emoción, expectativa, recuerdo, decisión... son conceptos que a menudo se usan de manera inestable o conceptos inestables. Pero, ¿qué se entiende por "conceptos inestables"?

Atribuimos "inestabilidad" a un objeto no firme: una mesa chueca o un compuesto químico que con facilidad cambia de estado ("esta muestra de azufre tiende a endurecerse y se transforma en la variedad rómbica"). También calificamos un carácter lábil de "inestable". Por analogía podemos reconstruir como "conceptos inestables" aquellos que frente a la reflexión, o a una reflexión que se prolonga en investigación científica:

a) son conceptos que poseen la tendencia a ser sustituidos por otros conceptos a partir de los cuales se producen descripciones diferentes y, por lo tanto, se obtienen explicaciones diferentes de los asuntos previamente conceptualizados; y

b) respecto de tales conceptos, encontramos dos formas básicas de sustitución. Hay inestabilidad homogénea si la sustitución de un concepto se lleva a cabo con conceptos capaces de recogerse por los puntos de vista de la primera o de la segunda persona. (El deseo, digamos, de atacar a una injusticia desde el punto de vista práctico de la primera persona se redescribe, 
desde el punto de vista práctico de la segunda persona, como una forma de resentimiento.) Hay inestabilidad heterogénea si para sustituir un concepto por otro se cambia de los puntos de vista de la primera o de la segunda persona exclusivamente al punto de vista de la tercera persona en alguno de sus diversos grados de radicalidad. (Por ejemplo, se pasa del punto de vista práctico de la primera o de la segunda persona al punto de vista en exclusiva teórico de la tercera persona: ataques a una injusticia o resentimientos se redescriben en lenguaje neurofisiológico.)

En las discusiones y procesos de explicación con frecuencia no encontramos estas formas puras de sustitución, sino combinaciones de ellas. Por otra parte, la inestabilidad conceptual no es un fenómeno raro. Por el contrario, es común. Día a día en las más diversas prácticas y con diversos intereses llevo a cabo ejercicios de ajuste y redescripción de mí mismo y de los otros animales humanos.

Quizá aclare vincular el contraste entre las dos formas básicas de inestabilidad conceptual con el contraste, más abarcador, entre actividad y pasividad. Hay inestabilidad homogénea cuando los conceptos presupuestos en la descripción de una acción se sustituyen por otros que implican actividad intencional por parte de una primera persona. Así, a veces sustituyendo la descripción de una acción por otra, reprocho: "Tu deseo no era casarte con ella; más bien, tenías la ambición de adquirir el prestigio social y la posición económica que traía consigo ese matrimonio." O bien: "En realidad tú no sientes celos de él, sino envidia y miedo y, tal vez, necesidad de castigarte."

No obstante, a menudo en las prácticas cotidianas se resbala a esas formas del punto de vista teórico de la tercera persona que, radicalizadas, constituyen el investigar científico. Por ejemplo, a veces se explica una acción no de acuerdo a las intenciones del agente, sino teniendo en cuenta su función efectiva - aunque no entendida por el agentedentro de un sistema social, tal como la podría reconstruir un sociólogo o un antropólogo: "Esa manifestación política, si bien no alcanzó a realizar ninguno de sus objetivos declarados, fue un éxito, pues reconstituyó la cohesión del grupo." A veces nos alejamos aún más del punto de vista de la primera persona, en particular cuando adoptamos el punto de vista de la investigación científica en ciencias naturales. Recordemos cuando un médico, produciendo inestabilidad conceptual ya heterogénea, corrige a su paciente: "Usted, más que tener deseos de huir del trabajo, se halla anémico, y a partir de su decaimiento tiene 
esas fantasías." O si un policía declara ante un tribunal: "Al no detenerse con semáforo en rojo, su manera de actuar se encontraba por completo fuera de control debido a las drogas que, inadvertidamente, le habían inyectado." En estos casos se sustituyen descripciones con conceptos intencionales ("tener deseos de huir del trabajo", "detenerse con semáforo en rojo") con descripciones con conceptos que implican algún grado de pasividad, de no intencionalidad, de parte de la primera persona.

No podemos ya, sin embargo, postergar ciertas dudas. ¿Qué nos aclara o explica tener en cuenta este uso de conceptos? Por ejemplo, ¿cuál es el tipo o tipos de dificultades que, por un lado, se pretende y, por otro, efectivamente se pueden tratar con conceptos inestables? De manera inmediata, tener en cuenta conceptos inestables parece resultar de la mayor utilidad en relación con una perspectiva normativa, si se quieren responder preguntas como: ¿De qué manera debo entender y explicar a las otras personas si no quiero ser injusto con ellas? ¿Qué puedo hacer con mi vida?

No resulta arduo comprobar que en las diversas circunstancias, so pena de resultar por completo parciales y lo que es peor, irresponsables, o hasta meramente ineptos por falta de discriminación, una y otra vez es necesario ajustar y reajustar normativamente los juicios a partir de las diversas formas de inestabilidad conceptual. Por eso, junto a las disculpas heterogéneas, las cautelas heterogéneas, los consejos heterogéneos, también a menudo consideramos conveniente formular preguntas heterogéneas como las siguientes: Cuando tu prima bebió tanto vino, ¿no previó que más tarde tendría que regresar a su casa manejando en la lluvia? Tu colega, ¿había alcanzado grados tan severos de adicción al tabaco que por ningún medio lo pudo dejar? Por otra parte, ¿acaso podemos planear con responsabilidad sin oscilar entre decisiones y predicciones, y corregir a las unas con las otras. Recuérdese las cautelas heterogéneas que adopta Ulises: sus zozobras sin duda pertenecen a un tipo de situaciones en el que —en tono menor- nos encontramos cada día.

Sin embargo, tener en cuenta la inestabilidad de ciertos conceptos, además de contribuir a responder preguntas normativas, o con dimensiones normativas, ¿ayudan también a responder la pregunta tal como se planteó al comienzo de esta reflexión: “¿quién es 'yo' "? Por lo pronto, podemos ya descomponer a esa pregunta inicial en dos subpreguntas:

1) La pregunta “¿quién es 'yo'?” proferida en actitud normativa de primera persona. 
O, si se prefiere, una expresión más común: “¿quién soy yo, al menos para mí?", conectando esta interrogación con preguntas como "¿qué debo hacer de mí mismo y cómo puedo hacerlo?", “¿cómo debo tratar a las otras personas?"

O también podemos preguntar: ¿quién es 'yo' desde el punto de vista de la tercera persona?

2) ¿Quién es 'yo', y punto?

Podemos reconstruir la pregunta 2) a partir de una perspectiva metodológica en exclusiva teórica. Así, para responderla, disponemos por lo menos de dos posibilidades:

2.1) Se postula que puede haber una teoría rigurosamente general y unitaria acerca de lo que hay. Se aceptan, pues, técnicas reductivistas fuertes - reductivistas "para todos los propósitos"- o eliminacionistas respecto de los deseos, creencias, emociones, recuerdos, expectativas, acciones..., en general, de todo el vocabulario mental y práctico. En este caso, si los razonamientos que he calificado como vértigos naturalistas fuesen, en realidad, razonamientos correctos, los conceptos inestables sólo servirían como conceptos pragmáticamente útiles para, en algunas circunstancias, ahorrarnos las complejas explicaciones correctas en términos de la biología, la química molecular o, tal vez, la física.

2.2) Se postula que no puede haber una teoría rigurosamente general y unitaria de lo que hay. De esta manera, se acepta que toda teorización y, en general, que todo uso del lenguaje se produce de acuerdo a intereses, con ciertos conceptos y, de esta manera, recortando algunos fragmentos y aspectos de la realidad y no otros. Según estas razones, de seguro se aceptará que las técnicas reduccionistas no son sólo útiles, sino condición necesaria de una gran cantidad de saberes y de tecnologías (respaldadas en ciencias naturales, pero también en ciencias como la psicología o la economía). No obstante, en cualquier caso se trata de técnicas reduccionistas débiles: establecen reducciones de algunas áreas respecto de otras, de acuerdo a algún o algunos intereses. No se trata, sin embargo, de procedimientos generales: de reducciones de cualquier área respecto de cualquier interés.

A partir de razones como éstas podrá defenderse que muchas de las diferentes teorizaciones - las teorías de la física, la química, la biolo- 
gía, la psicología, la sociología, la antropología, la economía, la lingüística, la ciencia política, o incluso reflexiones más o menos teóricas dependientes de saberes cualitativos como las teorizaciones sobre la música, la pintura, la gastronomía, la perfumería... - no se pueden o, más bien, no se deben concebir y trabajar como subteorías de una posible superteoría, capaz de unificarlas a todas. Por ejemplo, tal vez se razone que no tiene interés - no ilumina nada - unificar teorías químicas y teorizaciones literarias, o teorías físicas y teorías económicas; sin embargo, no hay por qué descartar que en muchas discusiones se encuentren subáreas productivas de intersección entre las teorías y teorizaciones en apariencia más dispares, como sugieren algunos tipos de inestabilidad conceptual. Pero se tratará sólo de algunas subáreas y con ciertos intereses particulares.

Quien acepte la respuesta 2.2 y las observaciones que la elaboran considerará que a los conceptos inestables no se los puede eliminar, ni a partir de una perspectiva normativa, ni de una metodológica. De esta manera, se defenderá que el fenómeno de la inestabilidad conceptual, en su versión tanto homogénea como heterogénea, forma parte también de la respuesta a la pregunta 2), que interroga "¿quién es 'yo'?"

Nuevas preguntas que no se dejan postergar: ¿contribuyen, entonces, los usos inestables de ciertos conceptos, los conceptos inestables, a defender una propuesta en la compleja y enredada polémica que se introdujo con las posiciones contrapuestas de Descartes y Hume? O más bien, ¿se procura con estos conceptos disolver esa polémica metafísica, o en parte metafísica, a la vez que se rescatan varias de sus inquietudes en polémicas normativas y metodológicas?

Volvamos a atender al concepto inestable de planear. Con frecuencia, para planear con responsabilidad, se tienen que poner en marcha procesos mixtos de deliberación, pues hay que incluir en el proceso de decidir, que inevitablemente pertenece al punto de vista de la primera persona, predicciones productos del punto de vista de la segunda o de la tercera persona, y viceversa. En consecuencia tendremos que situar, una y otra vez, el poder de la primera persona en un espacio ya demarcado por ciertos hechos (físicos, químicos, biológicos, psicológicos, sociales, políticos, económicos...).

¿Qué significa "sólo podemos planear algo con nosotros mismos... en un espacio ya demarcado por ciertos hechos"? Por lo pronto, otra manera de formular la creciente inquietud que hay detrás de las formas mixtas de comunicación y de uno de sus efectos, los conceptos inestables, consiste en hacer explícita una alternativa acerca de la posible génesis de la identidad personal, de la subjetividad, del yo: "O 
autoconstitución: el yo (la subjetividad, la identidad personal...) se encuentra en último término formado por el yo. O heteroconstitución: el yo es formado también — versión débil一 o sólo — versión fuerte- por antecedentes causales y funciones adaptativas propias de la evolución y de los procesos sociales de la historia (que son independientes del yo, del sujeto, de la subjetividad, de la identidad personal...)".

Sólo tiene en cuenta algunas razones, aunque no otras, quien para eliminar la inestabilidad conceptual y la contingencia procura defender la primera opción (lo que entiendo como "el vértigo de la primera persona", ese considerar que la primera persona es capaz de constituirse exclusivamente de acuerdo con sus decisiones) o la versión fuerte de la segunda opción (los vértigos naturalistas y sociales que eliminan o reducen el poder de la primera persona a un efecto entre otros de algún sistema biológico y/o social).

Consideremos una posible defensa de la primera opción, del vértigo de la primera persona, que indicase: las condiciones antecedentes y las funciones adaptativas de las acciones (tal como podrían reconstruirse en exclusiva desde el punto de vista de la tercera persona) no afectan de modo directo la actitud en primera persona, sino que dependen de lo que ésta planee hacer con ellas. Así, las condiciones antecedentes y las funciones adaptativas adquirirían sentido y valor, y también eficacia, sólo de acuerdo al modo en que la primera persona las asumiese en sus planes, o esbozos de planes. Esto no es verdad. Razones que no se tienen en cuenta en esa primera opción advierten que los significados que damos y cómo valoramos las condiciones antecedentes y las funciones adaptativas de las acciones depende, a su vez, de ciertas condiciones antecedentes y funciones adaptativas que la primera persona no planea: de las circunstancias y del carácter que sobreviene de -o es causado por, o es función, o emerge de...- la herencia biológica y las vicisitudes del sistema social en el que nos hallamos.

Por eso, hay que tener cuidado con palabras como "el modo de asumir los antecedentes causales y las funciones adaptativas en nuestros planes". No hay ningún plan abarcador que elija una primera persona que le subyazca como programa último. Tampoco hay una historia que sea para siempre "la historia de una vida". A las capacidades de seguimiento narrativo y planeador no les subyacen matrices unitarias que otorgan significado y valor a la vida de una persona. De ahí la incapacidad de "solidificarse" de cualquier seguir y seguirme. Una vez más se debe insistir: no existe una "cosa cartesiana", recóndita y fija, que constituya la primera persona fuera del traslape e interconexión de 
experiencias y acciones, o fragmentos de experiencias y acciones. Pero, ¿cómo puedo dar cuenta de procesos tan heterogéneos?

Precisamente, algunas de las dificultades que originan los vértigos simplificadores que acosan a quien se pregunta "¿quién soy yo?" —el vértigo naturalista, el social, el de la primera persona- pueden replantearse en torno a la expresión "proceso heterogéneo". Sospecha: tener en cuenta las formas heterogéneas de comunicación y el uso inestable de conceptos ino debilita las dificultades con que se nos presiona a partir de esa expresión?

Por lo pronto, ¿cómo dar cuenta de procesos tan heterogéneos como los que parecen presuponer palabras como "al constituirse en un cuerpo el sucederse de las identificaciones y contraidentificaciones sociales, en ese seguir y seguirse se va conformando una primera persona con cierto poder"? Obsérvese que en la pregunta anterior se combinan en un proceso varios materiales, a primera vista al menos, por completo heterogéneos. En primer lugar, encontramos el cuerpo humano: un organismo entre otros. En segundo lugar, tenemos los procesos que conforman las identificaciones y contraidentificaciones, productos de algunos sistemas sociales. En tercer lugar, topamos con - io postulamos?un poder que proviene de, o que hace intervenir y organiza estados mentales: el poder de la primera persona. Tanta real —io aparente?heterogeneidad no es casual que invite a absolutizar a cada uno de esos materiales. Así, suelen producirse argumentos que, según he procurado razonar un poco, resultan igualmente vertiginosos e igualmente simplificadores. Reitero: hay un vértigo naturalista que elimina el lenguaje práctico de la primera persona y sus descripciones y, con él, el vocabulario con que hacemos referencia a experiencias, acciones, deseos, creencias, emociones..., y lo sustituye con un lenguaje neurofisiológico, o químico, o físico. Por otra parte, hay un vértigo social en donde se reduce ese lenguaje de experiencias, acciones, deseos, creencias..., al lenguaje de los sistemas sociales, culturales, políticos, económicos. Pero también hay un vértigo del punto de vista de la primera persona que intenta independizar a las personas, en particular, todo aquello que corresponde a la actitud en primera persona, de sus antecedentes causales biológicos y sociales. ¿Qué podemos hacer frente a vértigos argumentales tan recurrentes como éstos?

Se propuso: se trata de operar de vez en cuando con conceptos inestables que nos recuerdan que las capacidades de seguimiento narrativo y planeador que conforman, al menos en parte, a la actitud en primer persona, sobrevienen -o son causadas, o son función, o emergendel funcionamiento de un tipo de cuerpo, el cuerpo humano. El cuer- 
po humano es un organismo con cierta vida biológica producto de la evolución. No hay vida biológica sin metabolismo. Disponer de metabolismo consiste en adquirir sustancias del medio ambiente - comiendo, bebiendo, respirando-y convertirlas en parte del propio cuerpo. En la naturaleza, el crecimiento y mantenimiento de un organismo depende de la adquisición de sustancias orgánicas e inorgánicas. Claramente, el metabolismo es un ejemplo característico de proceso heterogéneo. Si bien no se puede acceder al metabolismo desde el punto de vista de la primera persona (la primera persona no puede observar que su cuerpo absorbe las sustancias que consume), los efectos, al menos los negativos del metabolismo, se recogen a cada paso en las experiencias y las acciones de la primera persona: enseguida aprendo que no soy capaz de guardar ayuno por más de cierto tiempo, so pena de comenzar a perder fuerzas, o que desfallezco si regularmente no bebo líquidos. También pronto me di cuenta de que no debo mantenerme debajo del agua más de unos segundos.

Experiencias y acciones negativas como éstas suelen producir inestabilidad conceptual. Por otra parte, las formas mixtas de comunicación presuponen una dimensión del metabolismo que parece desbordar lo que suele entenderse con la expresión "vida biológica". Si lamento mis modales y los disculpo por una irritación provocada por la mala digestión, o me excuso por mi extrema parquedad a causa de la respiración entrecortada, de hecho reconozco que de algún modo el metabolismo convierte un pedazo de puerco en malos modales, agitación de la respiración en parquedad en mis respuestas. A su vez, se sabe: con malos y buenos modales, atención y desatención, se cultivan virtudes y vicios, responsabilidades e irresponsabilidades, se producen tradiciones, se afianzan comunidades, Estados, leyes, guerras... Por supuesto, entre el metabolismo biológico y lo que, metafóricamente, se califica como "metabolismo social" - las interrelaciones y retroalimentaciones entre los diferentes sistemas psicológicos, sociales, culturales, políticos, económicos, legales - hay, para abusar una vez más de esa palabra, numerosas "mediaciones".

\section{BIBLIOGRAFÍA}

Chalmers, D.J., The Conscious Mind: In Search of a Fundamental Theory, Oxford University Press, Oxford, 1996.

Churchland, Patricia, Neurophilosophy, A Bradford Book, The MIT Press, Cambridge, Mass., 1986.

Churchland, Paul, "Eliminative Materialism and the Propositional Attitudes", The Journal of Philosophy, vol. 78, 1981, pp. 67-90.

Diánoia, vol. LI, no. 57 (noviembre 2006). 
Descartes, R., Meditación segunda, en Meditationes de prima philosophia, ed. Charles Adam y Paul Tannery, Vrin, París, vols. VIII-IX, 1983. [Versión en castellano: Meditaciones metafísicas con objeciones y respuestas, trad. Vidal Peña García, Alfaguara, Madrid, 1977.]

— R. Descartes: Philosophical Letters, ed. A. Kenny, Clarendon Press, Oxford, p. 140.

Feyerabend, P., "Comment: Mental Events and the Brain", The Journal of Philosophy, vol. 60, no. 11, 1963, pp. 295-296.

Hume, D., A Treatise of Human Nature, ed. L.A. Selby-Bigge, Clarendon Press, Oxford, 1978.

MacIntyre, A., After Virtue, Duckworth, Londres, 1981.

Putnam, H., "The Meaning of 'Meaning'", en Mind, Language and Reality, Cambridge University Press, Cambridge, 1975, vol. 2, pp. 215-271.

Ricœur, P., Soi-même comme un autre, Édition Du Seuil, París, 1990.

Rorty, R., "Mind-Body Identity, Privacy, and Categories", The Review of Metaphysics, vol. 19, 1965, pp. 24-54.

Stich, S., From Folk Psychology to Cognitive Science: The Case Against Belief, The MIT Press, Cambridge, Mass.

Taylor, C., Sources of the Self, Cambridge, Cambridge University Press, 1989.

Recibido el 23 de febrero de 2006; aceptado el 4 de mayo de 2006. 